\title{
Controlled Nitric Oxide Release Using Poly(Lactic-Co-Glycolic Acid) Nanoparticles for Anti-Inflammatory Effects
}

Yoogyeong Oh', Hyejoong Jeong ${ }^{1}$, Sungmin Lim ${ }^{* 2}$ and Jinkee Hong ${ }^{*}, 1$

1 Department of Chemical \& Biomolecular Engineering, College of Engineering, Yonsei

University, 50 Yonsei-ro, Seodaemun-gu, Seoul 03722, Republic of Korea

2 Division of Cardiology, Department of Internal Medicine, Uijeongbu St. Mary's Hospital, College of Medicine, The Catholic University of Korea, Seoul, Korea.

${ }^{*}$ Correspondence to Jinkee Hong, Ph. D. (E-mail: jinkee.hong@yonsei.ac.kr), Sungmin Lim, M.D.Ph.D. (E-mail: sungmin@catholic.ac.kr) 
Supporting information

Figure S1: Preparation of BPEI/NONOate

Figure S2: Characterization of BPEI/NONOate

Figure S3: NO release profiles of BPEI/NONOate

Figure S4: TEM images of NO-PLGA

Figure S5: FT-IR spectra of NO-PLGA

Figure S6: Images of changes in emulsion stability over time

Figure S7: TEM images at low magnification of NO-PLGA after incubation for $24 \mathrm{~h}$

Figure S8: TEM images at high magnification of NO-PLGA after incubation for $24 \mathrm{~h}$

Figure S9: pH-responsive release of NO-PLGA 7525

Tabel S1: A summary of BPEI/NONOate's NO release profiles

Tabel S2: Elemental analysis of NO-PLGA 



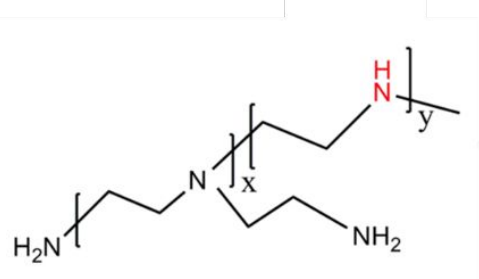

BPEI

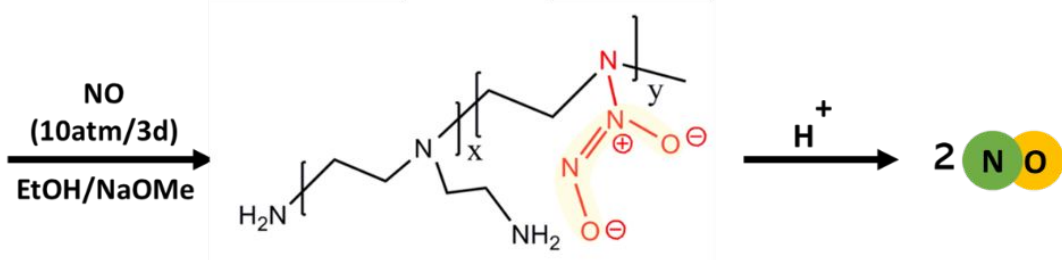

BPEI-N-diazeniumdiolate NO donor

Figure S1. Preparation of BPEI/NONOate

a

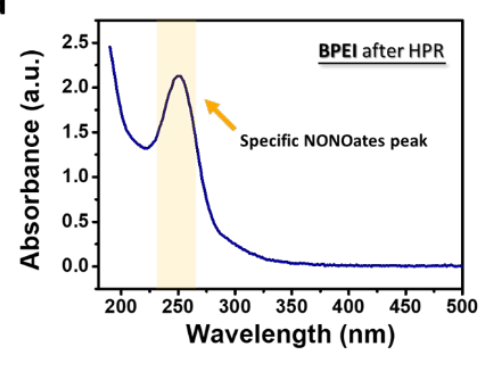

b

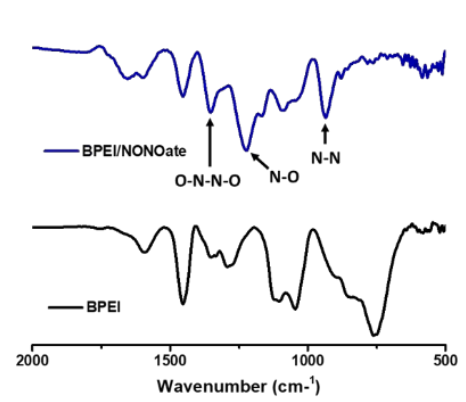

C

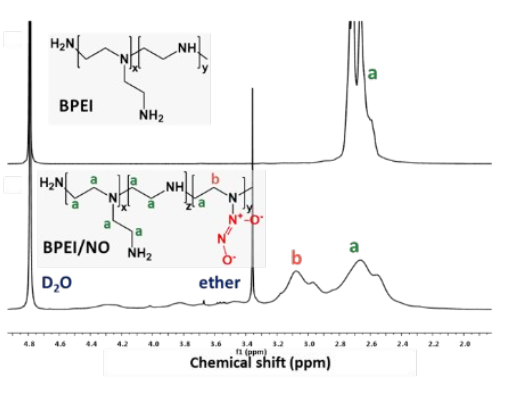

Figure S2. Characterization of BPEI/NONOate (a) UV-vis absorbance spectra, (b) FT-IR spectra and (C) $1 \mathrm{H}$ NMR spectra of BPEI/NONOate 

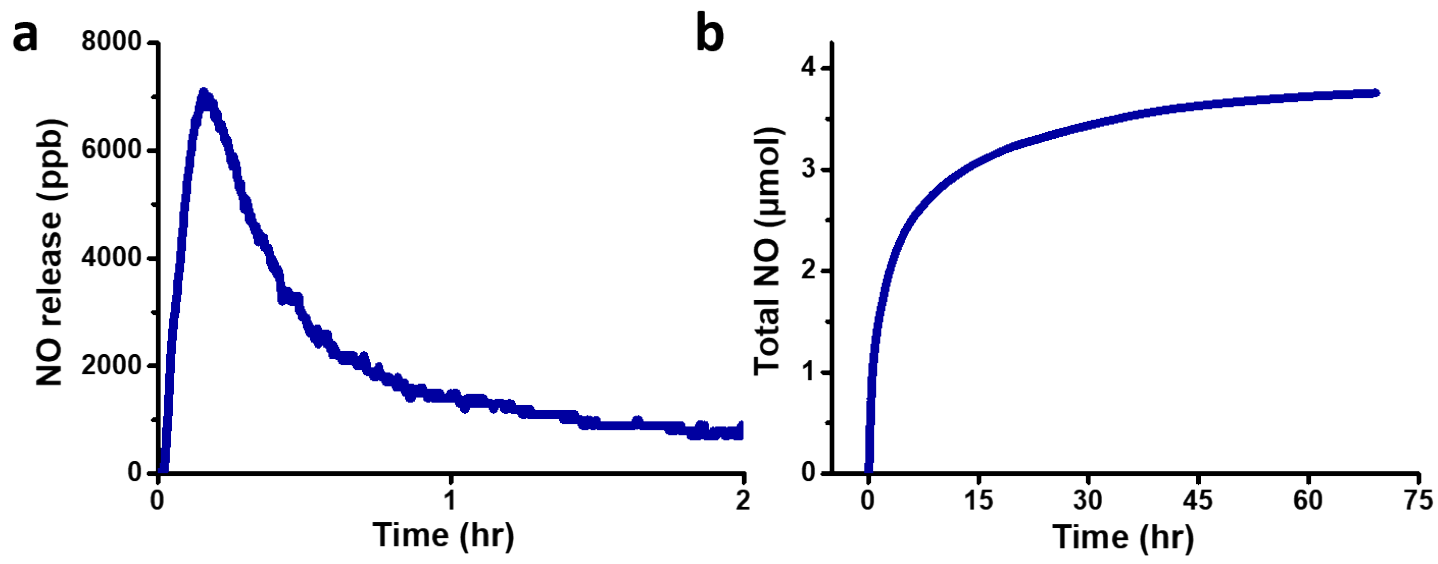

Figure S3. Nitric oxide release profiles of BPEI/NONOate in PBS $(1 \times, \mathrm{pH} 7.4)$ at $37^{\circ} \mathrm{C}$ :

(a) real-time NO release; (b) cumulative NO release.

Table S1. Nitric oxide release profiles of BPEI/NONOate

\begin{tabular}{l|l|l|l|l|l}
\hline & ${ }^{a} \mathrm{t}[\mathrm{NO}]$ & ${ }^{b} \mathrm{t}_{1 / 2}$ & ${ }^{\mathrm{c}}[\mathrm{NO}]_{\mathrm{m}}$ & ${ }^{\mathrm{d}} \mathrm{t}_{\mathrm{m}}$ & ${ }^{\mathrm{e}} \mathrm{t}_{\mathrm{d}}$ \\
\hline $\begin{array}{l}\text { BPEI/NONOat } \\
\mathrm{e}\end{array}$ & $3.76 \mu \mathrm{mol} \cdot \mathrm{mg}^{-1}$ & $2.45 \mathrm{~h}$ & $7100 \mathrm{ppb} \cdot \mathrm{mg}^{-1}$ & $7.25 \mathrm{~min}$ & $69.20 \mathrm{~h}$ \\
\hline
\end{tabular}

a t[NO] $\left(\mu \mathrm{mol} \cdot \mathrm{mg}^{-1}\right)$ : Total amount of $\mathrm{NO}{ }^{d} t_{d}(h)$ : Time duration release

${ }^{b} t_{1 / 2}(h):$ Half-life of NO e $t_{m}$ (min): Time until maximum flux of

${ }^{c}[\mathrm{NO}]_{\mathrm{m}}\left(\mathrm{ppb} \cdot \mathrm{mg}^{-1}\right):$ Maximum flux of $\mathrm{NO}$ Buffer: 1X PBS pH 7.4 
The NONOates formation was confirmed by the UV-vis, FT-IR, and NMR spectroscopies. In the UV spectrum, the maximum absorbance peak was observed at $250 \mathrm{~nm}$, in the characteristic NONOate wavelength range of $250-260 \mathrm{~nm}$ (Figure S2a). ${ }^{1}$ Furthermore, the BPEI/NONOate also exhibited a peak at 935,1223 , and $1352 \mathrm{~cm}^{-1}$, which are characteristic peaks for the $\mathrm{N}-\mathrm{O}$ stretches and $\mathrm{N}_{2}$ symmetric stretch of $\mathrm{N}_{2} \mathrm{O}_{2}{ }^{-}$ groups, respectively (Figure S2b). ${ }^{2}$ In ${ }^{1} \mathrm{H} N M R$, the proton signals of the methylene groups near the secondary amine sites of BPEl at $2.7 \mathrm{ppm}$ were shifted downfield to about 3.1 ppm in BPEI/NONOates as a result of the electron-withdrawing effect of the NONOates group (Figure S2c)..$^{1}$ Besides, NO release from BPEI/NONOates was analyzed in PBS solution at $37^{\circ} \mathrm{C}$. As shown in Figure S3, the initial burst release of NO was observed for 9 min, but NO release over 69 hours in a sustained manner (Table S1). 

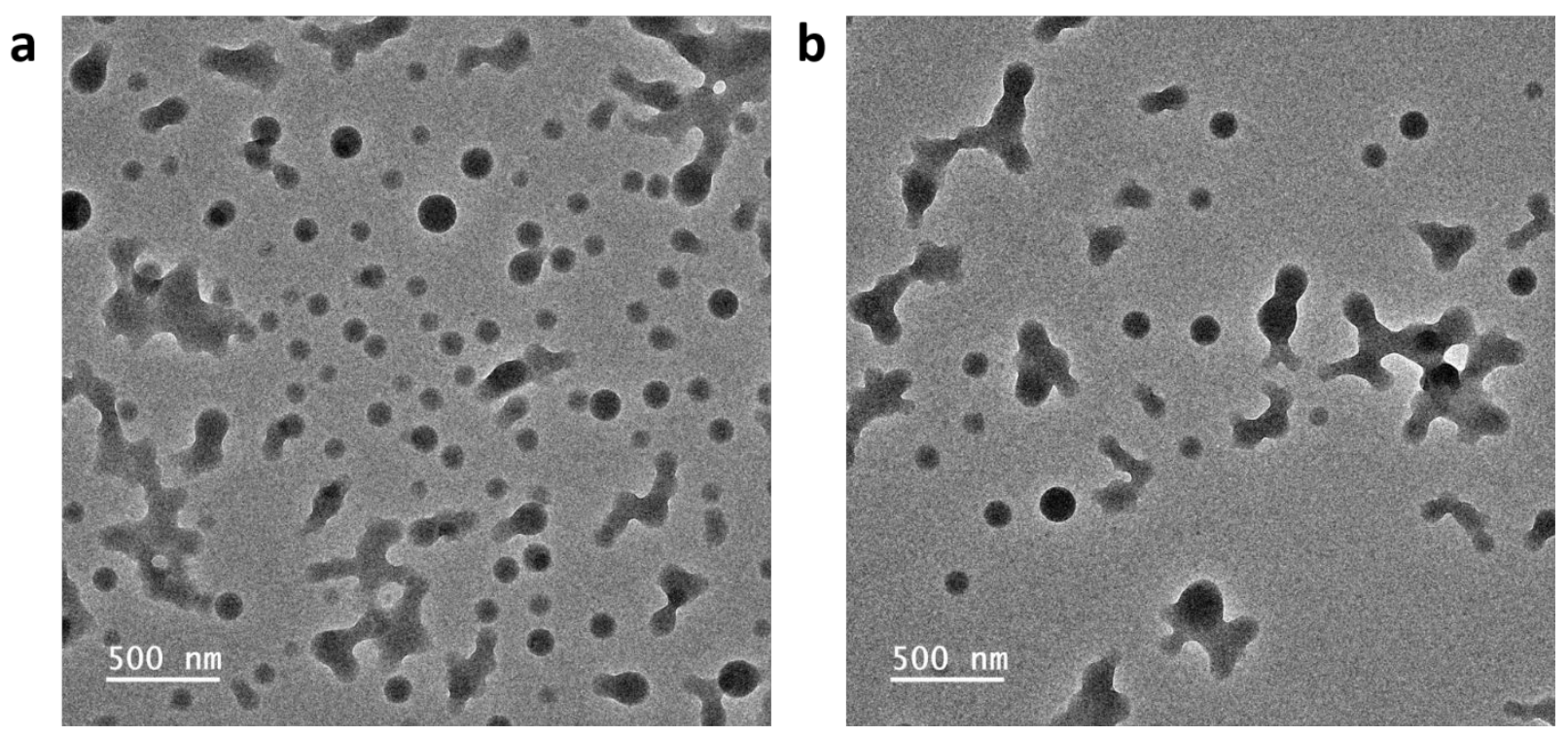

Figure S4 TEM images of NO-PLGA (a) PLGA 5050; (b) PLGA 7525

Table S2. XPS Atomic ratio of NO-PLGA surfaces

\begin{tabular}{lll}
\hline & NO-PLGA 5050 & NO-PLGA 7525 \\
\hline O1s & $38.42 \%$ & $38.08 \%$ \\
C1s & $59.16 \%$ & $60.36 \%$ \\
N1s & $2.42 \%$ & $1.56 \%$ \\
\hline
\end{tabular}




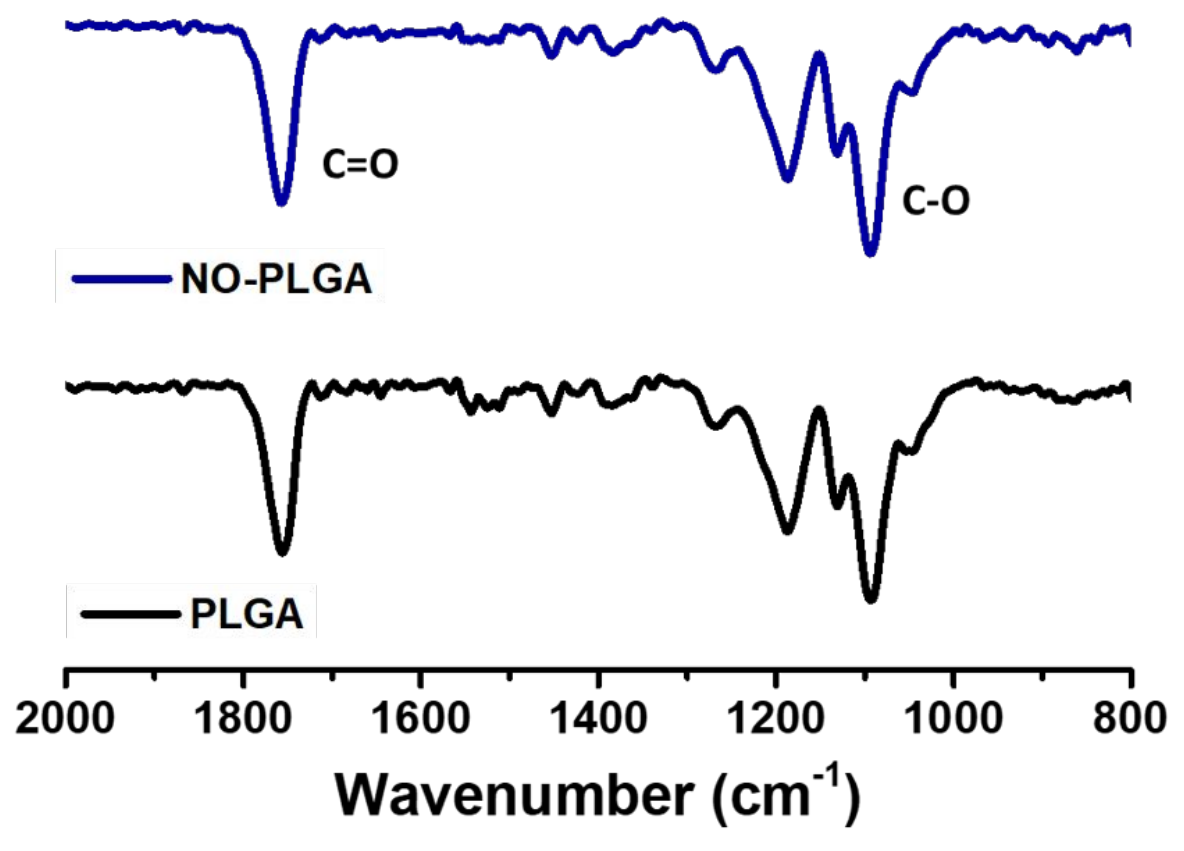

Figure S5. FT-IR spectra of NO-PLGA 

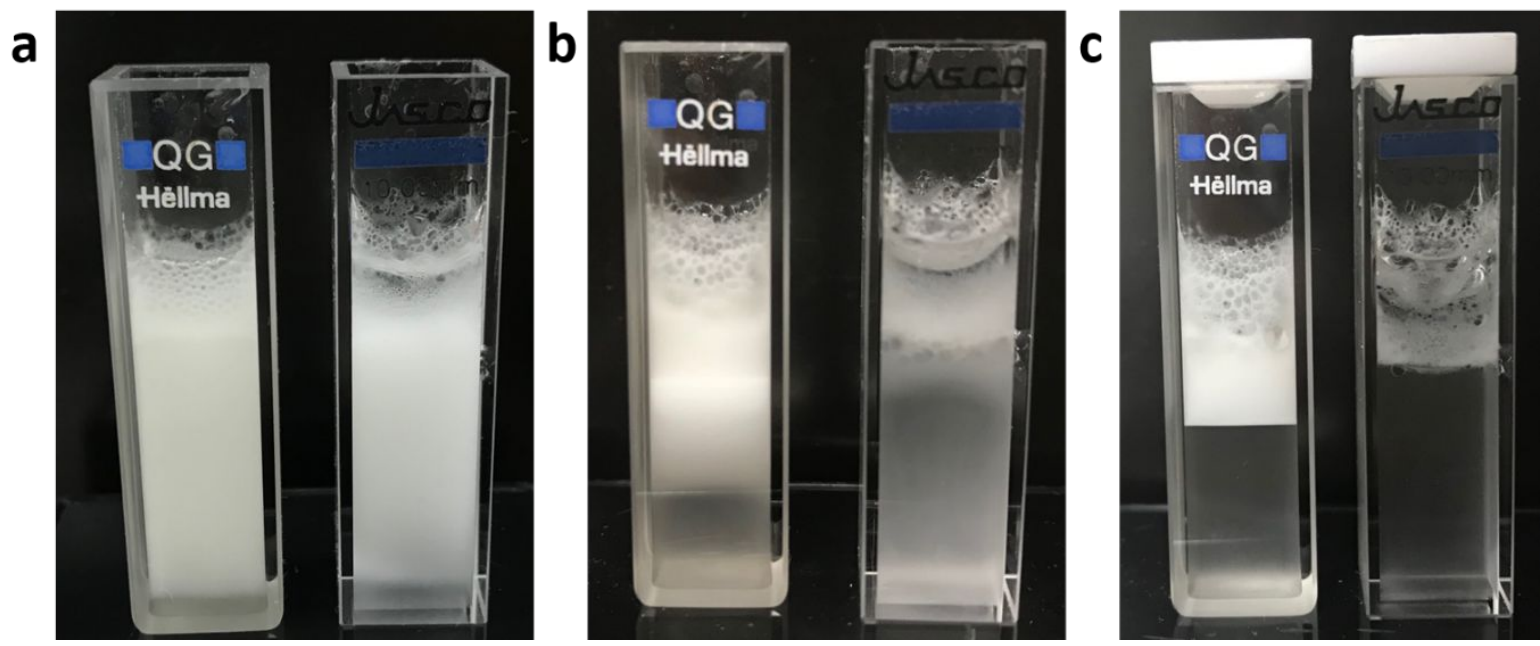

Figure S6. Changes in emulsion stability after (a) 100min, (b) 200min and (c) 500min.

Left side, PLGA 5050; Right side, PLGA 7525 


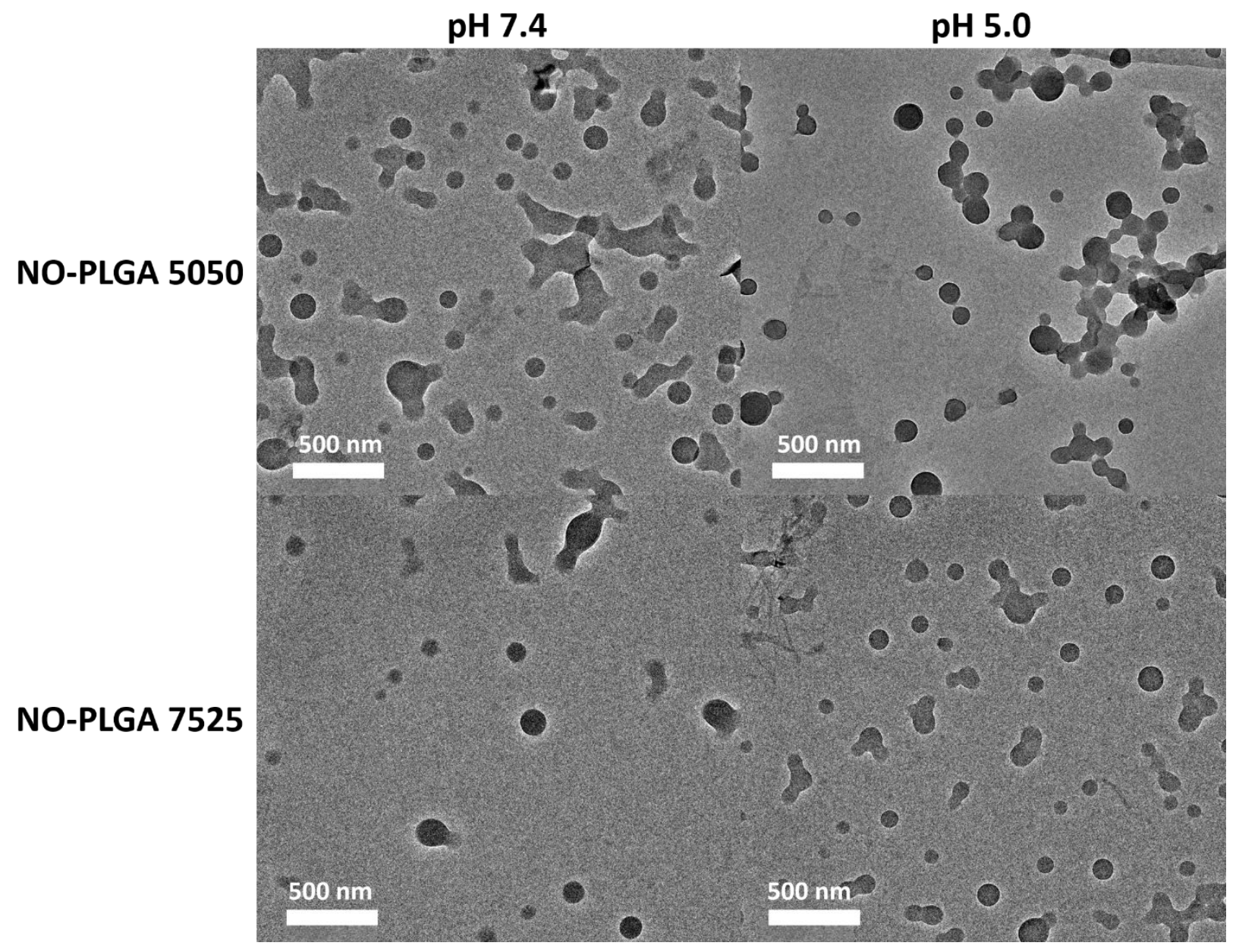

Figure S7. Changes in nanoparticle morphology. TEM images at low magnification of NO-

PLGA incubated in PBS (1×, pH 7.4) and sodium acetate buffer (100mM, pH 5.0) at $37^{\circ} \mathrm{C}$

for $24 \mathrm{~h}$ 


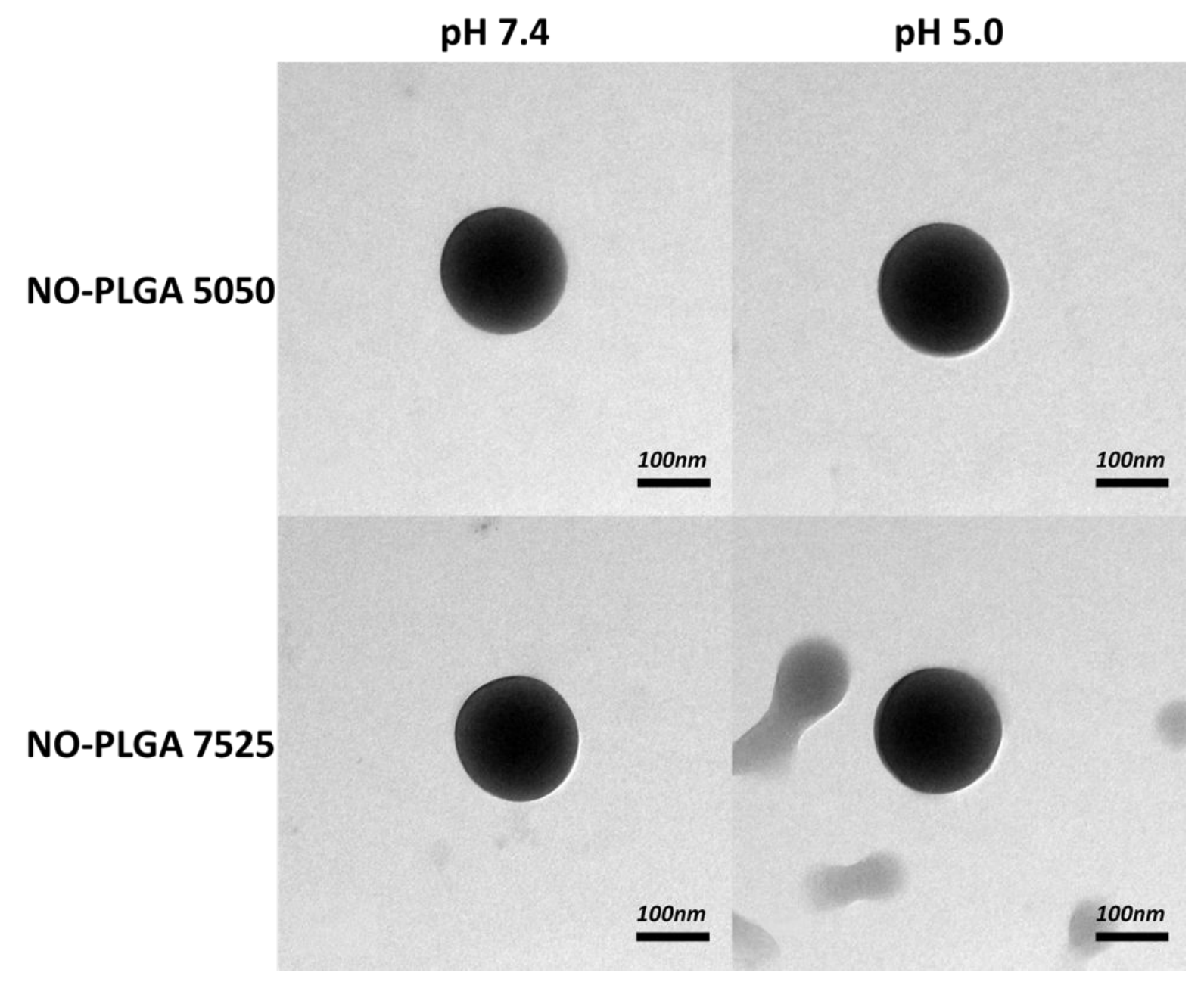

Figure S8. Changes in nanoparticle morphology. TEM images at high magnification of NO-PLGA incubated in PBS (1×, pH 7.4) and sodium acetate buffer (100mM, pH 5.0) at $37^{\circ} \mathrm{C}$ for $24 \mathrm{~h}$ 

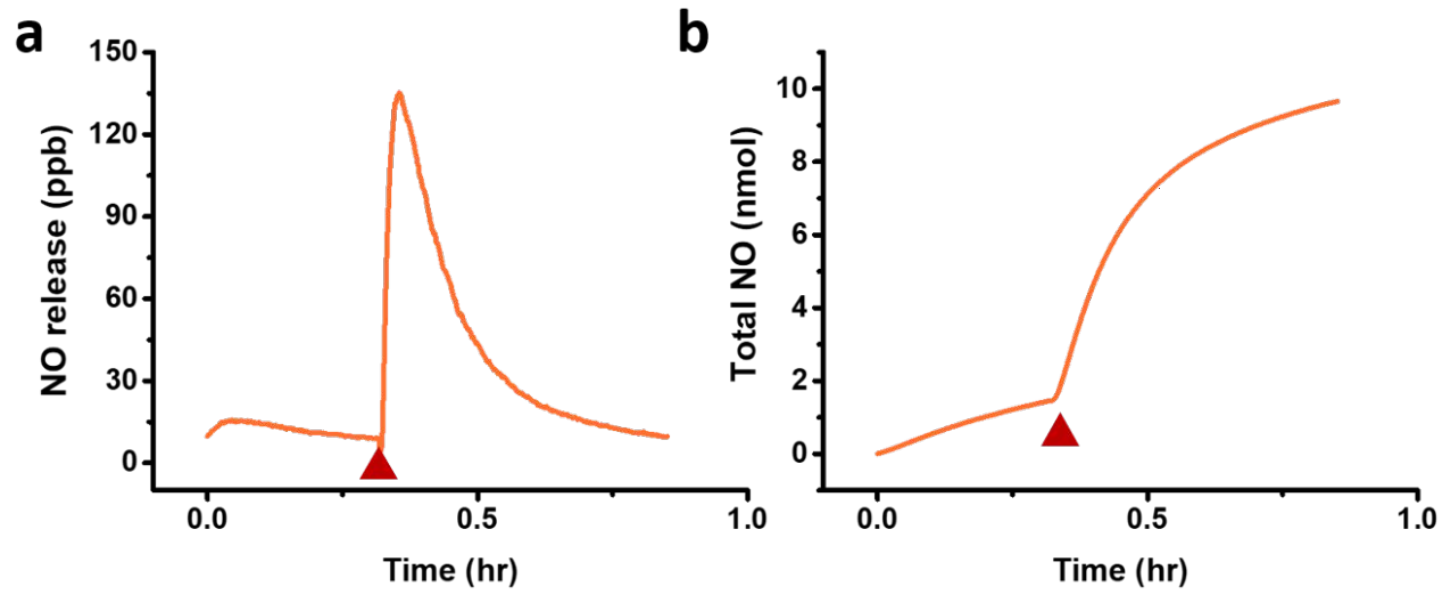

Figure S9. pH-responsive NO release of NO-PLGA 7525: (a) real-time NO release; (b)

cumulative NO release. NO-PLGA was immersed in PBS (1×, $\mathrm{pH} 7.4)$ and then acid was added for $\mathrm{pH}$-responsive $\mathrm{NO}$ release (acid; $1 \mathrm{M} \mathrm{HCl}$ ) (a red triangle; addition of acid) 


\section{REFERENCES}

1. Kim, J.; Lee, Y.; Singha, K.; Kim, H. W.; Shin, J. H.; Jo, S.; Han, D.-K.; Kim, W.

J., NONOates-polyethylenimine hydrogel for controlled nitric oxide release and cell proliferation modulation. Bioconjugate Chem. 2011, 22, 1031-1038.

2. Zhou, Z.; Annich, G. M.; Wu, Y.; Meyerhoff, M. E., Water-soluble poly (ethylenimine)-based nitric oxide donors: preparation, characterization, and potential application in hemodialysis. Biomacromolecules 2006, 7, 2565-2574. 